\title{
Composite Medium with Simultaneously Negative Permeability and Permittivity
}

\author{
D. R. Smith,* Willie J. Padilla, D. C. Vier, S.C. Nemat-Nasser, and S. Schultz \\ Department of Physics, University of California, San Diego, 9500 Gilman Drive, La Jolla, California 92093-0319
}

(Received 2 December 1999)

\begin{abstract}
We demonstrate a composite medium, based on a periodic array of interspaced conducting nonmagnetic split ring resonators and continuous wires, that exhibits a frequency region in the microwave regime with simultaneously negative values of effective permeability $\mu_{\mathrm{eff}}(\omega)$ and permittivity $\varepsilon_{\mathrm{eff}}(\omega)$. This structure forms a "left-handed" medium, for which it has been predicted that such phenomena as the Doppler effect, Cherenkov radiation, and even Snell's law are inverted. It is now possible through microwave experiments to test for these effects using this new metamaterial.
\end{abstract}

PACS numbers: 73.20.Mf, 41.20.Jb, 42.70.Qs

A periodic array of conducting elements can behave as an effective medium for electromagnetic scattering when the wavelength is much longer than both the element dimension and lattice spacing. What makes the resulting media special is that the effective permittivity $\varepsilon_{\text {eff }}(\omega)$ and permeability $\mu_{\text {eff }}(\omega)$ can have values not observed in ordinary materials. An example medium is a three-dimensional array of intersecting thin straight wires, for which propagating modes follow a dispersion relation analogous to that of a neutral plasma. This medium was initially used to study microwave propagation through the ionosphere $[1,2]$ [which exhibits a negative $\varepsilon_{\text {eff }}(\omega)$ below the plasma frequency], and has since been carefully reexamined and found to have further striking electromagnetic properties, including longitudinal plasmon modes [3-5].

In recent work [6], Pendry et al. have extended the range of electromagnetic properties of effective media by introducing a periodic array of nonmagnetic conducting units whose dominant behavior can be interpreted as having an effective magnetic permeability. By making the constituent units resonant, the magnitude of $\mu_{\mathrm{eff}}(\omega)$ is enhanced considerably, leading to large positive effective $\mu_{\text {eff }}(\omega)$ near the low frequency side of the resonance and, most strikingly, negative $\mu_{\mathrm{eff}}(\omega)$ near the high frequency side of the resonance.

The concept of negative $\mu_{\mathrm{eff}}(\omega)$ is of particular interest, not only because this is a regime not observed in ordinary materials, but also because such a medium can be combined with a negative $\varepsilon_{\text {eff }}(\omega)$ to form a "left-handed" material (i.e., $\mathbf{E} \times \mathbf{H}$ lies along the direction of $-\mathbf{k}$ for propagating plane waves). In 1968, Veselago [7] theoretically investigated the electrodynamic consequences of a medium having both $\varepsilon$ and $\mu$ negative and concluded that such a medium would have dramatically different propagation characteristics stemming from the sign change of the group velocity, including reversal of both the Doppler shift and Cherenkov radiation, anomalous refraction, and even reversal of radiation pressure to radiation tension. However, these effects could not be experimentally verified since, as Veselago pointed out, substances with $\mu<0$ were not available. Negative $\mu_{\text {eff }}(\omega)$ has been shown to be possible when a polariton resonance exists in the permeability, such as in the antiferromagnets $[8,9] \mathrm{MnF}_{2}$ and $\mathrm{FeF}_{2}$, or certain insulating ferromagnets [10]. However, a negative permeability with low losses coexisting with a negative $\varepsilon$ has not been demonstrated. The split ring resonator (SRR) medium recently introduced by Pendry et al. [6] has now given us the opportunity to make a material with negative permeability, from which a left-handed medium can be constructed, as we demonstrate below.

The system presented here is anisotropic to simplify the analysis; the isotropic medium, constructed by adding elements to increase the symmetry of a unit cell, will be presented elsewhere. A single copper SRR is shown in Fig. 1(inset) with the dimensions indicated. A time varying magnetic field applied parallel to the axis of the rings induces currents that, depending on the resonant properties of the unit, produce a magnetic field that may either oppose or enhance the incident field. The associated magnetic field pattern from the SRR is dipolar. By having splits in the rings, the SRR unit can be made resonant at

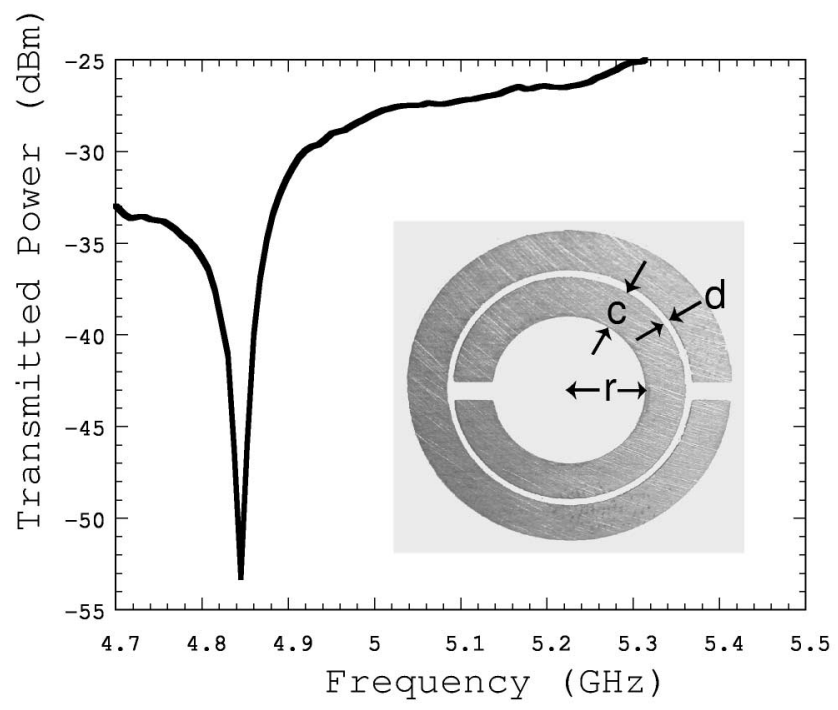

FIG. 1. Resonance curve of an actual copper split ring resonator (SRR). $c=0.8 \mathrm{~mm}, d=0.2 \mathrm{~mm}$, and $r=1.5 \mathrm{~mm}$. The SRR has its resonance at about $4.845 \mathrm{GHz}$, and the quality factor has been measured to be $Q=f_{0} / \Delta f_{3 d B}>600$, consistent with numerical simulations. 
wavelengths much larger than the diameter of the rings; that is, there is no half-wavelength requirement for resonance, as would be the case if the rings were closed. The purpose of the second split ring, inside and whose split is oriented opposite to the first, is to generate a large capacitance in the small gap region between the rings, lowering the resonant frequency considerably and concentrating the electric field. The individual SRR shown in Fig. 1(inset) has its resonance peak at $4.845 \mathrm{GHz}$. The corresponding resonance curve is shown in Fig. 1. Because the dimensions of the units are so much smaller than the free space wavelength, the radiative losses are small, and the $Q$ 's are relatively large.

By combining the split ring resonators into a periodic medium such that there is strong (magnetic) coupling between the resonators, unique properties emerge from the composite. In particular, because these resonators respond to the incident magnetic field, the medium can be viewed as having an effective permeability, $\mu_{\mathrm{eff}}(\omega)$. The general form of the permeability has been studied by Pendry et al. [6], where the following expression was derived:

$$
\begin{aligned}
\mu_{\mathrm{eff}} & =1-\frac{\pi r^{2} / a^{2}}{1-3 \ell / \pi^{2} \mu_{0} \omega^{2} C r^{3}+i\left(2 \ell \rho / \omega r \mu_{0}\right)} \\
& =1-\frac{F \omega^{2}}{\omega^{2}-\omega_{0}^{2}+i \omega \Gamma} .
\end{aligned}
$$
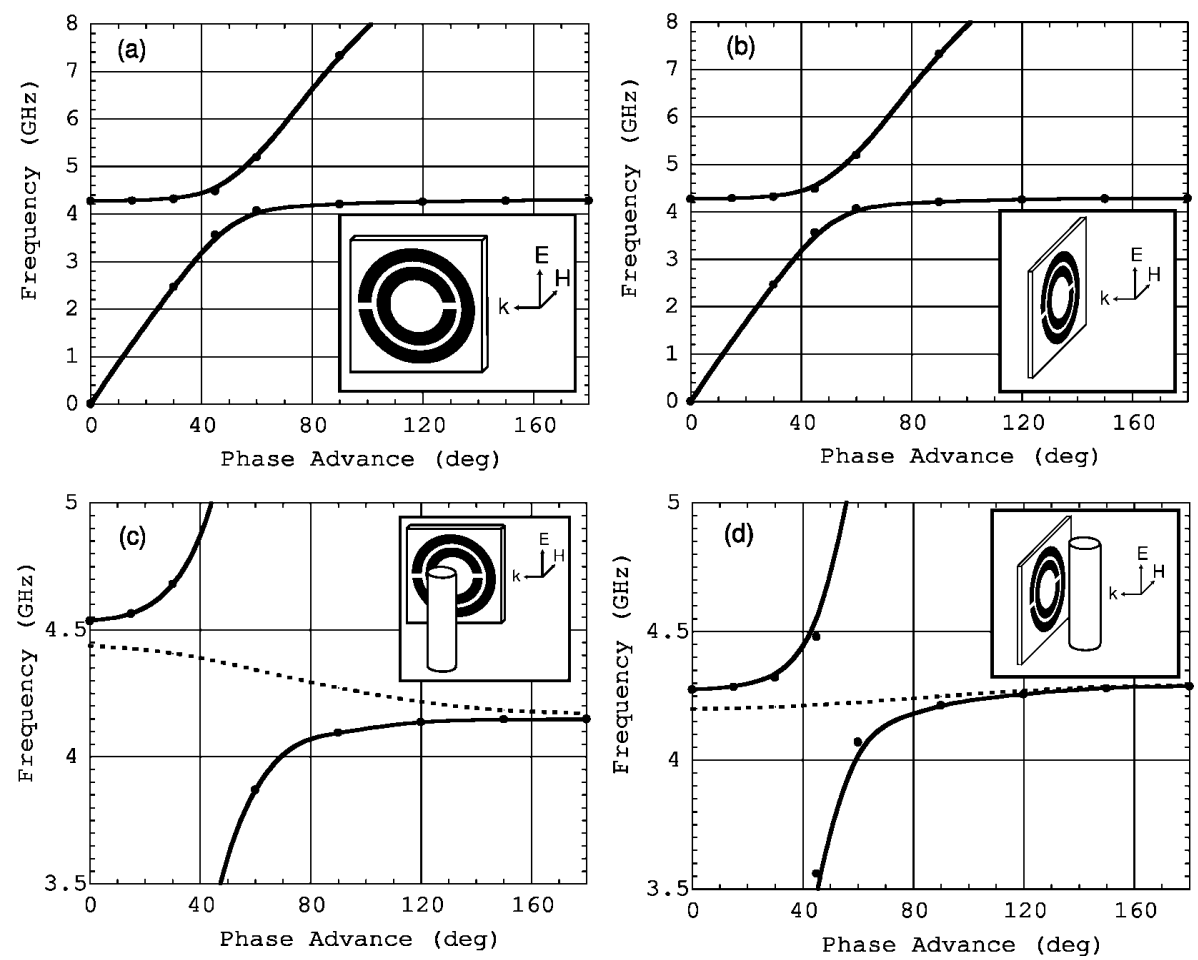

FIG. 2. (a) Dispersion curve for the parallel polarization $H_{\|}$. The lines with the solid circles correspond to the split ring resonators only. The inset shows the orientation of the split ring with respect to the incident radiation. The horizontal axis is the phase advance per unit cell, or $k d$, where $k$ is the wave number. (b) Dispersion curve for the perpendicular polarization $H_{\perp}$. The lines with the solid circles correspond to the split ring resonators only. The inset shows the orientation of the split ring with respect to the incident radiation. (c) Expanded view of the dispersion curve shown in (a). The dashed line corresponds to the split ring resonators with wires placed uniformly between split rings. (d) Expanded view of the dispersion curve shown in (b). The dashed line corresponds to the split ring resonators with wires placed uniformly between split rings. The insets to (c) and (d) show the orientations of the split rings with respect to the wires. 
resonance in the $\varepsilon_{\text {eff }}(\omega)$ with constant $\mu_{\text {eff }}(\omega)$ or due to a resonance in $\mu_{\text {eff }}(\omega)$ with constant $\varepsilon_{\text {eff }}(\omega)$, which we have indicated is the case. We discuss below the method we use to demonstrate that there is a region of negative $\mu_{\mathrm{eff}}(\omega)$.

Using MAFIA [11], a commercial electromagnetic mode solver, dispersion curves were generated for the periodic infinite metallic structure consisting of the split ring resonators. There are two incident polarizations of interest: magnetic field polarized along the split ring axes $\left[H_{\|}\right.$, Fig. 2(a), inset], and perpendicular to the split ring axes [ $H_{\perp}$, Fig. 2(b), inset]. In both cases, the electric field is in the plane of the rings. As shown by the curves in Figs. 2(a) and 2(b), a band gap is found in either case, although we will show that we can interpret the $H_{\|}$gap of Fig. 2(a) as being due to negative $\mu_{\text {eff }}(\omega)$ and the $H_{\perp}$ gap of Fig. 2(b) as being due to negative $\varepsilon_{\text {eff }}(\omega)$. The negative permeability region for the $H_{\|}$modes begins at $4.2 \mathrm{GHz}$ and ends at 4.6 GHz, spanning a band of about $400 \mathrm{MHz}$. Not evident from the figure, but consistent with the model indicated in Eq. (1), $\mu_{\text {eff }}(\omega)$ switches to a large negative value at the lower band edge, decreasing in magnitude (but still negative) for increasing frequency through the gap. At the upper band edge, $\mu_{\text {eff }}(\omega)=0$, and a longitudinal mode exists (not shown), identified as the magnetic plasmon mode by Pendry et al. [6]. For the dielectric gap shown in Fig. 2(b), the same behavior is observed, but with the roles of $\varepsilon_{\text {eff }}(\omega)$ and $\mu_{\text {eff }}(\omega)$ reversed.

Short of performing an intricate reflection and transmission measurement to fit the material constants, there is no simple means to deduce whether the split ring medium is responding electrically or magnetically. We can, however, use a physical approach and alter the dielectric function of the surrounding medium, creating scattering properties that can distinguish whether the band gaps are due to either the $\mu_{\text {eff }}(\omega)$ or $\varepsilon_{\text {eff }}(\omega)$ of the SRR being negative.

In a $2 \mathrm{D}$ medium composed of periodically placed conducting straight wires, there is a single gap in propagation up to a cutoff frequency, $\omega_{p}$, for modes with the electric field polarized along the axis of the wires [1-5]. This onset of propagation has been identified with an effective plasma frequency dependent on the wire radius and spacing, with the effective dielectric function following the form $\varepsilon_{\text {eff }}(\omega)=1-\omega_{p}^{2} / \omega^{2}$. A reduction in $\omega_{p}$ can be achieved by restricting the current density to the thin wires, which also increases the self-inductance per unit length, $L$ [3-5]. When the conductivity of the wires is large, the plasma frequency has been shown to have the general form $\omega_{p}=\left(d^{2} L \varepsilon_{0}\right)^{-1 / 2}$, and thin wire structures can easily be made to have $\omega_{p}$ at microwave or lower frequencies. Combining the SRR medium having a frequency band gap due to a negative permeability with a thin wire medium produces a resultant left-handed material in the region where both $\mu_{\text {eff }}(\omega)$ and $\varepsilon_{\text {eff }}(\omega)$ have negative values.

Numerical simulations were carried out in which parallel wires of radius $0.8 \mathrm{~mm}$ were added in between the split rings, in a direction parallel to the incident electric field as shown in the inset of Fig. 2(c). The results of these simu- lations are shown as dashed lines in Figs. 2(c) and 2(d). For the wires alone, a gap extends from zero frequency to $\omega_{p}$, at $13 \mathrm{GHz}$. When wires are added symmetrically between the split rings, for the $H_{\|}$case a passband occurs within the previously forbidden band of the split ring dispersion curves of Fig. 2(a). That this passband [the dashed line in Fig. 2(c)] occurs within a previously forbidden region indicates that the negative $\varepsilon_{\mathrm{eff}}(\omega)$ for this region has combined with the negative $\mu_{\mathrm{eff}}(\omega)$ to allow propagation, as predicted.

By combining the ideal frequency dependence for the wire medium [3] with Eq. (1) for the permeability of split rings, we can derive the following expression for the dispersion relation of the combined medium:

$$
k^{2}=\frac{\left(\omega^{2}-\omega_{p}^{2}\right)}{c^{2}} \frac{\left(\omega^{2}-\omega_{b}^{2}\right)}{\left(\omega^{2}-\omega_{0}^{2}\right)} .
$$

This equation shows that the range of the propagation band ( $k$ real) extends from $\omega_{0}$ to $\omega_{b}=\omega_{0} / \sqrt{1-F}$. This was formerly the region of the gap of the SRR structure in the absence of the wires. Note that the dispersion relation leads to a band with negative group velocity everywhere, and a bandwidth that is independent of the plasma frequency (provided $\omega_{p}>\omega_{b}$ ).

The behavior of the magnetic gap can be contrasted with that occurring for the $H_{\perp}$ case, which we have identified as a dielectric gap. Because $H$ is parallel to the plane of the SRR, we assume magnetic effects are small, and that $\mu_{\text {eff }}(\omega)$ is small, positive, and slowly varying. As shown in Fig. 2(d), a passband again occurs, but now outside of the forbidden region, and within a narrow range that ends abruptly at the band edge of the lowest propagation band. The passband in this case occurs where the effective dielectric function of the split rings exceeds the negative dielectric function of the wire medium. As the dispersion curves calculated do not include losses, there will always be a range of passband frequencies, however narrow, when the resonant dielectric medium of split rings is combined with the negative dielectric medium of wires. Once again, we can describe the behavior of the dielectric gap by an approximate dispersion relation,

$$
k^{2} \simeq \frac{\left(\omega^{2}-\omega_{p}^{2}\right)}{c^{2}} \frac{\left(\omega^{2}-\omega_{f}^{2}\right)}{\left(\omega^{2}-\omega_{0}^{2}\right)},
$$

where $\omega_{f}^{2}=\omega_{0}^{2} \omega_{p}^{2} /\left(\omega_{0}^{2}+\omega_{p}^{2}\right)$. In deriving Eq. (3), we have neglected the difference between $\omega_{0}$ and $\omega_{b}$, as $\omega_{b}$ does not play an essential role here, and assumed $\omega_{p} \gg \omega_{0}$. The propagation band in this case extends from $\omega_{f}$ to $\omega_{0}$, with a bandwidth strongly dependent on the plasma frequency. As the plasma frequency is lowered, the lower edge of the propagation band lowers, increasing the overall bandwidth. The group velocity of this band is always positive. Both Eqs. (2) and (3) neglect material losses (i.e., $\Gamma=0$ ). The contrast between the two propagation bands in the $H_{\|}$and $H_{\perp}$ cases illustrates the difference between the magnetic and dielectric responses of the split ring medium. 


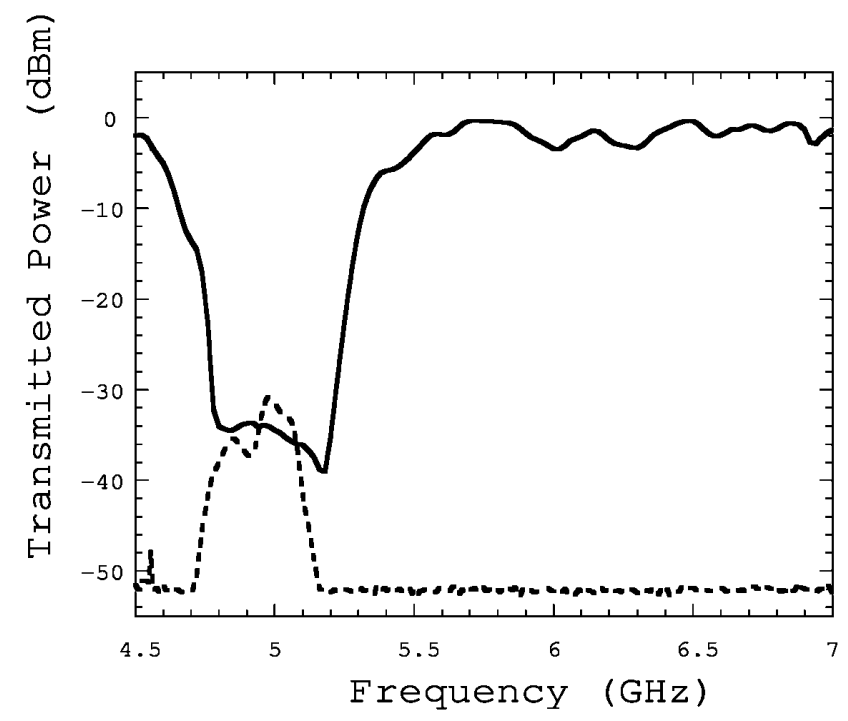

FIG. 3. A transmission experiment for the case of $H_{\|}$. The upper curve (solid line) is that of the SRR array with lattice parameter $a=8.0 \mathrm{~mm}$. By adding wires uniformly between split rings, a passband occurs where $\mu$ and $\varepsilon$ are both negative (dashed curve). The transmitted power of the wires alone is coincident with that of the instrumental noise floor $(-52 \mathrm{~dB})$.

The SRRs such as that shown in Fig. 1(inset) were fabricated using a commercially available printed circuit board. In order to test the results of the simulations, square arrays of SRRs were constructed with a lattice spacing of $8.0 \mathrm{~mm}$ between elements. The resonant mode of an individual element resembles a magnetic dipole, with electric fields strongly confined to the gap region between the rings, and the magnetic field circulating around the rings as in a wire loop. As the magnetic flux generated by the SRR is required to return within the unit cell, the fractional area $F$ is the critical parameter for the enhancement of the permeability.

Microwave scattering experiments were performed on the fabricated SRR medium, and the combined SRR/metal wire medium. In order to ease the required size of the structure, we utilized a two-dimensional microwave scattering chamber, discussed previously in detail [12]. The scattering chamber is made out of aluminum, with a grid pattern of holes in the top plate to allow source and probe antenna coupling. Microwave absorber material placed around the periphery of the chamber minimized reflection back into the scattering region.

For the $H_{\|}$polarization we constructed 17 rows of split rings in the $H$ direction (eight elements deep in the propagation direction), oriented as in Fig. 2(a), inset. Figure 3 shows the results of transmission experiments on split rings alone (solid curve), and split rings with wires placed uniformly between (dashed curve). The square array of metal wires alone had a plasma frequency of $12 \mathrm{GHz}$; the region of negative $\varepsilon$ below this frequency attenuated the transmitted power to below the noise floor of the microwave detector $(-52 \mathrm{dBm})$. When split rings were added to the wire array, a passband occurred, consistent with the propagation region indicated by the simulation [Fig. 2(c)].

We have demonstrated both by numerical simulation and experiment that an effective medium of only conducting elements responds predominantly to the magnetic field of incident electromagnetic fields. Remarkably, there is a band of frequencies for which $\mu_{\mathrm{eff}}(\omega)$ can be negative, here manifested as a region of attenuation in scattering from a finite section of material. We have combined this material with a negative $\varepsilon_{\text {eff }}(\omega)$ material to form a "left-handed" medium, forming a propagation band with negative group velocity where previously there was only attenuation. We are now in a position to further investigate the fascinating electrodynamic effects anticipated for such composite metamaterials.

The authors thank Professor John Pendry for introducing them to the special properties of the SRR and the effective negative $\mu(\omega)$ concept as well as to helpful unpublished results and discussions. They also thank Mike Wiltshire of G. E. C. Marconi for supportive comments and discussion. This work was supported by the NSF (Contracts No. NSFDMR-96-23949 and No. NSF-DMR-97-24535) and the DOE (Contract No. DOE-DE-FG-03-93ER40793).

*To whom all correspondence should be addressed. Electronic address: drs@sdss.ucsd.edu

[1] R. N. Bracewell, Wireless Engineer (Iliff \& Sons Ltd., London, 1954), p. 320.

[2] W. Rotman, IRE Trans. Antennas Propag. AP10, 82 (1962).

[3] J. B. Pendry, A. J. Holden, W. J. Stewart, and I. Youngs, Phys. Rev. Lett. 76, 4773 (1996).

[4] J. B. Pendry, A. J. Holden, D. J. Robbins, and W. J. Stewart, J. Phys. Condens. Matter 10, 4785 (1998).

[5] D. R. Smith, D. C. Vier, Willie Padilla, Syrus C. NematNasser, and S. Schultz, Appl. Phys. Lett. 75, 1425 (1999).

[6] J. B. Pendry, A. J. Holden, D. J. Robbins, and W. J. Stewart, IEEE Trans. Microwave Theory Tech. 47, 2075 (1999).

[7] V. G. Veselago, Sov. Phys. USPEKHI 10, 509 (1968).

[8] D. L. Mills and E. Burstein, Rep. Prog. Phys. 37, 817 (1974).

[9] R.E. Camley and D.L. Mills, Phys. Rev. B 26, 1280 (1982).

[10] A. Hartstein, E. Burstein, A. A. Maradudin, R. Brewer, and R. F. Wallis, J. Phys. C 6, 1266 (1973).

[11] The MAFIA Collaboration, Release 4.0, F. Ebeling et al., MAFIA User Guide, 1996.

[12] D. R. Smith, R. Dalichaouch, N. Kroll, S. Schultz, S. L. McCall, and P. M. Platzman, J. Opt. Soc. Am. B 10, 314 (1993). 